\title{
Séance du Comité central du 14 février 2013
}

\begin{abstract}
Loi sur l'enregistrement des maladies oncologiques Dans le cadre de la procédure de consultation concernant la loi sur l'enregistrement des maladies oncologiques, le Comité central (CC) déplore la nouvelle procédure qui prévoit que ce soit aux médecins traitants de collecter les données, ce qui occasionnerait une charge administrative importante et inutile. Il regrette également que l'Office fédéral de la statistique (OFS) obtienne plus d'informations que le registre national et demande une clarification du rôle de l'OFS et des autres services concernés ainsi qu'une distinction systématique entre registres cliniques et registres épidémiologiques.
\end{abstract}

Institut pour l'informatique au cabinet - En prévision d'une future collaboration avec l'Institut pour l'informatique au cabinet (IPI), le CC a décidé de mettre sur pied un groupe de travail conjoint, chargé de rédiger un document de discussion succinct à l'intention de l'Assemblée des délégués (AD).
Présidence de l'Assemblée des délégués - Le poste de président de l'Assemblée des délégués doit être repourvu d'ici à fin 2013 au plus tard. Pour une transition optimale, l'élection doit avoir lieu cet été. Les sociétés faîtières sont dès lors priées d'annoncer leurs éventuels candidats.

Parité des données et loi sur les registres - Dans le cadre de ce projet, le groupe de travail 22a du domaine Données, démographie et qualité (DDQ) a six mois pour développer un concept qui devrait être présenté à l'AD cet automne.

Loi sur les épidémies - Suite au dépôt du référendum sur la loi relative aux épidémies, la FMH et l'Association suisse des infirmières et des infirmiers (ASI) ont décidé de publier un communiqué de presse commun. Les deux acteurs doivent encore décider s'ils poursuivront leur collaboration dans le cadre de la campagne de votation.

\section{Nouvel outil pédagogique:} manuel de formation au cabinet médical.

\section{Ouvrage didactique pour formateurs et assistantes médicales en formation.}

